Journal Wetenskap Health

\title{
Complications and Diseases in the Postpartum and Their Treatment
}

\author{
Johana Timbe \\ College of Health Sciences, University of Nairobi, Kenya
}

\begin{abstract}
The general purpose of the study is to know and understand the basic concepts of presentation and position at birth and dystocia during labor. The infection that happened causes complicatons and disease for example Mother's milk dam, breast infection, Tromboplebitis, and Psychological disorders during Postpartum. Postpartum period which starts from the time the baby is born and the placenta is born until the womb returns to what it was before pregnancy. The paper will discusse about the symtoms, system and treatment of the disease in the Postpartum.
\end{abstract}

Keywords: Complications, Postpartum, Physicological Disorders

\section{Introduction}

The puerperium or post partum period is the period after delivery is completed up to 6 weeks or 42 days. After the puerperium period, the reproductive organs will gradually experience changes like before pregnancy. During the postpartum period, it needs more attention because the $60 \%$ maternal mortality rate occurs during the puerperium. The maternal mortality rate (MMR) is the cause of the number of women dying from a cause of lack of attention to post partum women (Maritalia, 2012). In developing countries, the postpartum period is a critical period for mothers who have given birth. It is estimated that $60 \%$ of maternal deaths occur after delivery and $50 \%$ of them occur within the first 24 hours (Praeirardjo, 2006). The high rate of postpartum mortality is a complex problem that is difficult in a country. If MMR is still high, it means that obstetric services are still poor, so that the need for mothers is high, namely 420 per 100,000 live births compared to other ASEAN countries. We estimated maternal mortality at the global, regional, and national levels from 1990 to 2015 for ages $10-54$ years by systematically compiling and processing all available data sources from 186 of 195 countries and territories, 11 of which were analysed at the subnational level. We quantified eight underlying causes of maternal death and four timing categories, improving estimation methods since GBD 2013 for adult all-cause mortality, HIV-related maternal mortality, and late maternal death. Secondary analyses then allowed systematic examination of drivers of trends, including the relation between maternal mortality and coverage of specific reproductive health-care services as well as assessment of observed versus expected maternal mortality as a function of Socio-demographic Index (SDI), a summary indicator derived from measures of income per capita, educational attainment, and fertility (Kassebaum et al 2016)

\section{Definition}

\section{Endometritis}

According to Satue et al. (2016) the most common type of infection is enometritis, where germs enter the endometrium, usually in the placental inertial scar, and in a short time involve the entire endometrium. In infections with less pathogenic bacteria, the inflammation is limited to the endometrium. The decidual tissue together with the blood clot becomes necrotic and secretes a smelly sap and consists of necrotic crumbs and fluid. At the boundary 
between the inflamed area and the healthy area there is a layer consisting of leukocytes. In more severe infections the endometrial boundary can be crossed and spread occurs

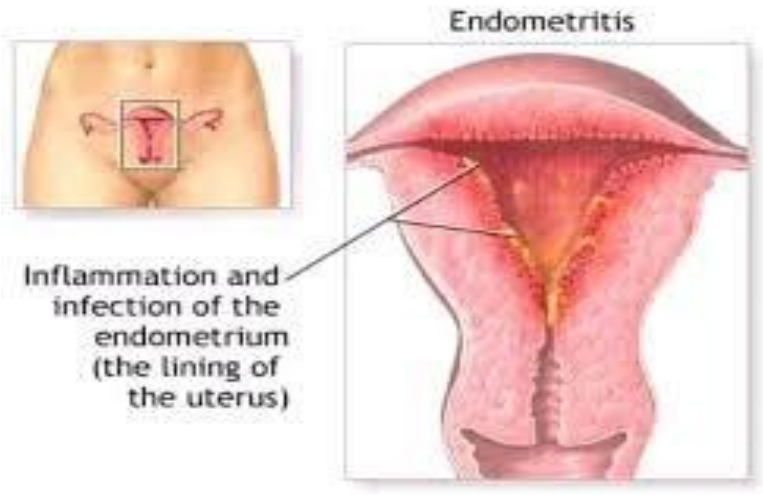

Figure 1. leukocytes

\section{Epidemiologi}

(1) After vaginal delivery, the incidence is $1-3 \%$ of all vaginal deliveries. (2) After cesarean section delivery, the incidence is $13-90 \%$ of all caesarean section deliveries. (3) In the non-obstetric population (who are not pregnant), endometritis can coexist with other infections such as salpingitis, oophoritis and pelvic peritonitis, the incidence is $70-90 \%$.

\section{Risk factor}

The risk increases when: (1) Miscarriage (2) Childbirth, especially after prolonged or cesarean delivery (3) Medical procedures that involve entering the uterus through the cervix such as hysteroscopy (4) Placement of an intrauterine device (IUD) (4) Dilation and curettage (scrapping of the uterus).

The risk does not increase with: (1) Age (2) Race (3) Family planning pills (4) Use of condoms (5) Sex during menstruation.

\section{Etiology}

Usually involves 2-3 infectious agents asending, curettage, endometrial biopsy, hysteroscopy, IUD, prolonged labor, cesarean delivery, and miscarriage. (1) Gonorrhea (2) Ureaplasma urealyticum (3) Peptostretococcus (3) The B streptococcus group (4) Bacteroides bivius (5) Gardnerella vaginalis (6) Chlamydia = late onset postpartum endometritis (7) Enterococcus $=$ in $25 \%$ of women receiving cephalosporin therapy (8) Herpes = rare (9) Tuberculosis $=$ rare.

\section{Symptoms and Signs}

(1) Swelling of the stomach (2) Abnormal bleeding from the vagina (3) Abnormal discharge from the vagina / discharge (4) Fever (5) Restlessness, feeling sick, general condition is bad (6) Abdominal or pelvic pain due to uterine pain.

\section{Pathology}

(1) Endometrial or decidual infection usually occurs ascending (2) In acute endometritis $=$ there are neutrophils in the endometrial gland (3) In chronic endometritis $=$ there are plasma cells and lymphocytes in the endometrial stroma (4) Chronic endometritis in the obstetric population $=$ associated with postnatal conception or selective abortion (5) Chronic endometritis in nonobstetric population $=$ associated with infections (chlamydia, tuberculosis, bacterial vaginosis) and uterine contraceptives. 


\section{Pathophysiology}

(1) Spread of infection from the endometrium to the fallopian tubes, or peritoneal cavity can cause salpingitis, oophoritis, local peritonitis, or tubo-ovarian abscess. (2) Salpingitis can cause tubal dysmotility and adhesion dysfunction, resulting in infertility, increased risk of ectopic pregnancy, and chronic pelvic pain.

\section{Governance}

(1) Broad-spectral antibiotics (clindamycin and IV gentamicin every 8 hours) $=90 \%$ of women will improve within 48-72 hours (2) Intravenous fluids \& hospital bed rest = complex cases (after delivery).

\section{Complications}

(1) Infertility (2) Other pelvic infections (3) Peritonitis (4) Pelvic or uterine abscess (5) Pelvic hematoma (6) Bacteremia (7) Septic shock.

\section{Peritonitis}

According to Ghali et al. (2011) puerperal peritonitis can occur due to widespread endometritis, but it can also be seen together with salpingo-oophoritis and pelvic cellulitis. Furthermore, it is possible that the abscess in pelvic cellulitis oozes pus into the peritoneal cavity and causes peritonitis.

\section{Symptoms}

(1) Sufferers with fever (2) Lower abdominal pain, but in good general condition (2) Pelvioperitonitis may present an abscess growth. The pus that normally collects in the douglas cavity should be drained with a posterior colpotomia to prevent its passage through the rectum or bladder.

\section{Cause}

(1) The temperature has risen to high (2) Pulse fast and small (3) Abdominal bloating and pain, there are muscular defenses (4) The face of the patient, which at first becomes red, becomes pale, the eyes are sunken, the skin is cold (5) There is what is called the hippocratica facies (6) High general peritonitis mortality.

\section{Handling}

(1) Perform nasogastric tube insertion if flatulence is due to ileus (2) Give $3000 \mathrm{ml}$ infusion (NaCL or Ringer's lactate) (3) Give antibiotics so that they are free of heat for 24 hours: Ampicillin $2 \mathrm{~g} \mathrm{IV}$, then $1 \mathrm{~g}$ every 6 hours, plus Gentasmicin $5 \mathrm{mg} / \mathrm{kg}$ body weight IV single dose / day and metronidazole $500 \mathrm{mg}$ IV every 8 hours (4) Laparotomy is required for cleaning the stomach (peritoneal lavage) if there is an abscess bag.

\section{Mother's Milk Dam}

Abnormal conditions in the breast, generally occur due to blockage of the milk ducts or because the breasts are not completely emptied (Mahoney et al. 2014).. This happens a lot in mothers who give birth for the first time. Dams of breast milk can occur because the breasts are not emptied, because the mother feels unfamiliar with breastfeeding and is afraid of nipple blisters when breastfeeding. The role of midwives in accompanying and providing knowledge on lactation at this time is very much needed and of course midwives must be very patient in assisting breastfeeding mothers to continue breastfeeding their babies. 


\section{Symptoms of a breast milk dam}

(1) Swelling, pain throughout the breast or local pain (2) Redness of the entire breast or only local (3) Hard and lumpy breasts (crouching) (4) Heat and general pain.

\section{Management}

(1) Continued breastfeeding. First the baby is fed on the affected breast for as long and as often as possible, so that the breast is empty. Then to normal breasts. If the mother is unable to empty the breast by breastfeeding her baby, then emptying is done by pumping the milk (2) Give a hot compress, when using a warm shower or hot wet cloth on the affected breast (3) Change the position of breastfeeding from time to time, ie lying down, sitting or holding the ball (football position) (4) Wear clothes with a loose bra (5) Get adequate rest and eat nutritious foods (6) Drink lots of about 2 liters per day. Through the application of methods like the above, usually the inflammation will disappear after 48 hours, rarely becomes an abscess. However, if the above methods do not improve after 12 hours, then antibiotics are given for 5-10 days and analgesics.

\section{Breast Infections}

During the puerperium, infection and inflammation can occur in the breasts, especially in the primiparous. Signs of infection are chills accompanied by an increase in temperature, the patient feels lethargic and there is no appetite, the cause of infection is staphylococcus aureus. The cause of infection is staphylococcus aureus. Mammae was enlarged and painful and somewhere, the skin was red, swollen slightly and painful on the touch. If not treatment an abscess can occur

\section{Cause}

Breast infections are usually caused by bacteria that are found on normal skin (Staphylococcus aureus). Bacteria often come from the mouth of the baby and enter the milk ducts through tears or cracks in the skin (usually on the nipples). Mastitis usually occurs in women who are breastfeeding and most commonly occurs within 1-3 months after delivery. About 1-3\% of breastfeeding women develop mastitis in the first few weeks after giving birth. The causes of breast infection; (1) Swollen breasts that are not fed adequately (2) Bra that is too tight (3) Putting milk blisters that cause infection (4) Poor nutrition, insufficient rest and anemia.

\section{Symptoms}

(1) Swelling and pain (2) Breasts appear red all over or in a certain place (3) Breasts feel hard and lumpy (4) There is fever and general pain.

\section{Prevention}

Nursing care of nipples in lactation is an important attempt to prevent mastitis. The treatment consists of cleaning the nipples with baby oil before and after breastfeeding to remove crusts and dry milk. Besides that, it also helps mothers who breastfeed their babies to be infection-free with staphylococus. If there is a wound or crack in the nipple, it is better if the baby does not breastfeed the breast in question, and milk can be drained by massage.

\section{Treatment}

As soon as mastitis is discovered, the baby's milk feeding is stopped and given the following treatment: (1) Give cloxacillin $500 \mathrm{mg}$ every 6 hours for 10 days (2) Breast support (3) Cold compress (4) If necessary give paracetamol $500 \mathrm{mg}$ orally every 4 hours (5) Follow progress 3 days after administration of treatment. Handling and Role of Midwives : (1) Breast 
compressed with warm water (2) To reduce pain analgesic treatment can be given (3) To treat infection given antibiotics (4) The baby starts feeding from the inflamed breast (5) Instruct the mother to always breastfeed her baby (6) Instruct the mother to eat nutritious food and get enough rest.

\section{Thromboplebitis}

Thromboplebitis is the invasion or expansion of pathogenic microorganisms that follow the bloodstream along the veins and their branches. Thromboplebitis preceded by thrombosis, can occur in pregnancy but is more often found during the puerperium (Roos, 2014).

\section{Etiology}

Several factors cause thromboplebitis: (1) Changes in blood structure (2) Changes in circulatory rateInjury to the internal lining of blood vessels. During pregnancy and especially in childbirth when the placenta is released, the levels of fibrinogen which plays an important role in blood clotting increase, making it easier for clots to occur.

\section{Symptoms}

The symptoms of this disease include: (1) Swelling and redness (2) Pain to the touch and a warm sensation in the touched area. When the vein is near the surface of the skin. When a vein in the leg is affected standing or walking. Symptoms of this disease can also be accompanied by fever. However, many people with venous thrombosis have no symptoms. When you see that the vessels appear hard, red, swollen, or sore, seek medical care immediately. Especially if a person's occupation allows immobility or if there is a family history of thromboplebitis. Emergency medical nurses should be sought if the symptoms are severe and accompanied by shortness of breath or high fever, which may be a condition of DVT, which can increase the risk of blood clots especially to the lungs.

\section{Perineal Wounds; Post Partum Bleeding; Stages: Primary and Secondary \\ Primary Postpartum Bleeding}

Primary postpartum hemorrhage, namely postpartum bleeding that occurs within the first 24 hours of birth. The main causes of primary postpartum hemorrhage are uterine antonychia, retained placenta, remaining placenta, torn birth canal and uterine inversion.

\section{Secondary Postpartum Bleeding}

Secondary postpartum hemorrhage is bleeding that occurs after 24 hours of delivery, less than 6 weeks after delivery.

\section{Causes of uterine antonias, placental retention, and birth canal tears}

(1) Antonia uteri, It is the failure of the myometirum to contract after childbirth so that the uterus is fully relaxed, widened, flaccid and unable to perform the occlusion function of blood vessels. The result of this uterine Antonia is bleeding. Bleeding in uterine antonias comes from blood vessels that are open at the mark adhesion of the placenta which is partially or completely detached (2) Placenta Retention, Placental retention is a condition that has not been born half an hour after the fetus is born. This is caused by: (1) The placenta has not separated from the uterine wall (2). The placenta is separated, but not yet born. If the placenta has not separated at all there will be no bleeding, but if part of the placenta is removed. The placenta has not separated from the uterine wall due to: (1).Uterine contractions are not strong enough to release the placenta (placenta adhesiva) (2) The placenta is firmly attached to the uterine wall because the corial villi penetrate the decidua to the myometrium (placenta accreta) (3). The placenta is firmly attached to the uterine wall 
because the corial villi penetrate below the peritoneum (placenta percreta). The placenta has separated from the uterine wall but has not yet come out, due to the absence of attempts to give birth or due to mismanagement of the third stage, resulting in a circle of contraction in the lower part of the uterus that blocks the passage of the placenta (placental incarceration).

\section{Birth Canal Tear}

Tears of the birth canal can occur together with Antonia uteri. Postpartum bleeding with a well-contracted uterus is usually caused by cervical or vaginal tears. after delivery, the vulva and perineum should always be examined. Examination of the vagina and cervix with a speculum also needs to be done after delivery (Vlasyuk, 2019). Tears of the birth canal always give bleeding in varying amounts. Bleeding originating from the birth canal should always be evaluated, namely the source and amount of bleeding so that it can be treated. Sources of bleeding can come from the perineum, vagina, cervix, and uterine tears (uterine rash). Bleeding can be in the form of a hematoma and tear of the birth canal with arterial bleeding or rupture of a vein. To be able to determine the source of bleeding can be done by deep examination and examination of the speculum after the source of bleeding is known with certainty, bleeding is stopped by performing ligation (Malvasi et al. 2014)

\section{Psychological disorders during childbirth}

Postpartum Depression, Postpartum depression is when a mother feels sadness, guilt, and other common forms of depression for a long time after giving birth. Causes of postpartum depression (1) Physical changes: after childbirth there are very large hormonal changes in the female body (especially the female hormones estrogen and progesterone) (2) Physical problems: having a baby (especially for the first time) often causes the mother to be anxious and insecure about her ability to care for the newborn.

\section{Complications of postpartum depression}

(1) For mothers: postpartum depression that is left untreated and can last up to months (2) For fathers: postpartum depression can have lasting effects in the family (3) For children: children whose mothers experience postpartum depression may have emotional and behavioral disorders, such as eating and sleeping disorders.

\section{Postpartum Depression Treatment}

(1) Psychotherapy. A session used for the mother and perhaps to be able to meet with a psychiatrist or psychologist, then discuss all the issues that underlie depression (2) Antidepressant drugs. Antidepressant drugs can be given by a doctor if needed.

\section{Treatment of postpartum depression}

(1) During pregnancy, the doctor will observe the symptoms and signs of depression. During pregnancy some mothers have a tendency to have mild depression (2) After the baby is born. After the baby is born the doctor will recommend routine checks to find out if there are signs of depression or baby blouse syndrome.

\section{Postpartum Blues}

Behavioral symptoms: (1) Often cry (2) Hyperactivity / often excessive (3) Too sensitive (4) Easy to take offense (5) Don't care for babies. Physical symptoms: (1) Lack of sleep (2) Loss of energy (3) Loss of appetite / increased appetite (4) Easily tired after waking up. Emotional symptoms: (1) Excessive physical anxiety and worry (2) Confused (3) Excessive physical worry (4) Not confident (5) Sad (6) Feelings of being ignored. 


\section{Postpartum Psychosis}

Postpartum psychosis symptoms: (1) Hearing voices and seeing things that are not there (hallucinations) (2) Extreme mood changes (mood swings) (3) Feel disconnected from reality (4) Feeling confused may not recognize friends and family (5) Imagining, believing in things that are not true or not logical.

\section{Conclusion}

Birth is the process by which the fetus and membranes are pushed out through the birth canal. The post partum period is a period of six weeks from the time the baby is born until the reproductive organs return to their normal pre-pregnancy state. This period is sometimes called the puerperium or fourth trimester of pregnancy. The physiological changes that occur are very clear, even though they are considered normal, in that the pregnancy processes are reversed. Partus partum is the process of expulsion of the fetus that occurs at term pregnancy with the provisions of the mother or without advice or drugs. It is hoped that midwives will be able to have more knowledge about post partum as well as the ability to process data and perform management in patients with post partum. With the right knowledge, it is hoped that the provision of nursing care will be carried out effectively and will not experience problems in the midwifery nursing care process.

\section{References}

Ghali, J. R., Bannister, K. M., Brown, F. G., Rosman, J. B., Wiggins, K. J., Johnson, D. W., \& McDonald, S. P. (2011). Microbiology and outcomes of peritonitis in Australian peritoneal dialysis patients. Peritoneal Dialysis International, 31(6), 651-662.

Kassebaum, N. J., Barber, R. M., Bhutta, Z. A., Dandona, L., Gething, P. W., Hay, S. I., ... \& Lopez, A. D. (2016). Global, regional, and national levels of maternal mortality, 1990-2015: a systematic analysis for the Global Burden of Disease Study 2015. The Lancet, 388(10053), 1775-1812.

Mahoney, M. C., \& Ingram, A. D. (2014). Breast emergencies: types, imaging features, and management. American Journal of Roentgenology, 202(4), W390-W399

Malvasi, A., Tinelli, A., Barbera, A., Eggebø, T. M., Mynbaev, O. A., Bochicchio, M., ... \& Di Renzo, G. C. (2014). Occiput posterior position diagnosis: vaginal examination or intrapartum sonography? A clinical review. The Journal of Maternal-Fetal \& Neonatal Medicine, 27(5), 520-526.

Maritalia, D., \& Nifas, B. A. K. (2012).

Roos, K. L. (2014). Acute bacterial infections of the central nervous system. In Aminoff's Neurology and General Medicine (pp. 795-815). Academic Press.

Satué, K., \& Gardon, J. C. (2016). Infection and infertility in mares. Genital Infections and Infertility.

Vlasyuk, V. V. (2019). The Concept of Birth Trauma. In Birth Trauma and Perinatal Brain Damage (pp. 17-21). Springer, Cham. 